\title{
Die Taetigkeit der Kampange Gegen Homofobie fuer gesselschaftliche Bewegung Lesbian, Gay, Bisexual, Transgender (LGBT) in Polen
}

\author{
JANUSZ JARTYŚ \\ DR \\ Uniwersytet Szczeciński, Wydział Humanistyczny \\ janujar.eu@gmail.com
}

Keyword social movement, LGBT, Poland, human rights

Abstract The activity of Campaign Against Homophobia constitutes a crucial element of the LGBT social movement in Poland. It is directed towards non-heterosexual people and for society in general. In this article the author analyze the activity of $\mathrm{CAH}$, pointing the legal and psychological help especially for LGBT movement and educational activity among polish society. It has to be underlined, that the analyzed organization in this article, deals with pro-health and educational polices and has great success in defending the rights of LGBT people in Poland. Worth noting is the publishing activity which consist of periodical reports of LGBT social situation in Poland.

Działalność Kampanii Przeciw Homofobii (KPH) na rzecz ruchu społecznego Lesbian, Gay, Bisexsual, Transgender (LGBT) w Polsce

Słowa kluczowe ruch społeczny, LGBT, Polska, prawa człowieka

Abstrakt

Działalność Kampanii Przeciw Homofobii stanowi istotny element funkcjonowania ruchu społecznego LGBT w Polsce. W niniejszym artykule autor dokona analizy działalności kampanii, wskazując pomoc prawną i psychologiczną dla osób LGBT oraz edukacyjną udzielaną polskiemu społeczeństwu. Należy podkreślić, że organizacja będąca przedmiotem analizy w niniejszym artykule prowadzi szeroko pojętą politykę prozdrowotną oraz edukacyjną, ma na swoim koncie także sukcesy w obronie praw osób LGBT w Polsce. Na uwagę zasługuje ponadto prowadzona działalność wydawnicza, wśród której istotny element stanowią wydawane cyklicznie raporty, obrazujące sytuację społeczną osób LGBT w Polsce.

Der grosse politisch-gesselschaftlicher Wandel nad dem Jahr 1989 in Polen sorgte dafuer, dass in der Oeffentlichkeit tauchten solche Probleme auf, die bis jetzt als Tabutehmen bezeichnet 
wurden, Eines von ihnen beschaeftigt sich mit den Rechten der Menschen, die unter LGBT ${ }^{1}$ existtieren und deren Rechte in der Oeffentlichkeit artikuliert werden.Die Bewegung LGBT, die sich in Polen schrittenweise entwickelte, nahm in den neunziger Jahren verschiedene Institutionsformen an. Man kann hier zum Beispiel regionale Vereine "Lambda" nennen, die unter anderen in Stettin (Szczecin), Warschau (Warszawa), oder Krakau (Kraków) taetig waren. Aber erst das Interesse der linken Parteeien an den Problemen der Nichtheterosexuellen und die Entwicklung des Internets nach der XX und XXI Jahrhundertwende haben dazu beigetragen, dass die Forderungen der LGBT Bewegung zum gesellschaftlichen Bewusstsein gelangen. Eine gewisse Rolle hat hier, die im Jahre 2001 Kampagne Gegen Homofobie (KPH) gespielt - gesamtpolitische Organisation gemeienmuetziger Koerperschaft die fuer die Nichtheterosexualle handelt (Kampania Przeciw Homofobii, 2016). In der angenommenen Bezeichnung hat sie an die Angst gegen LGBT Pesonen angeknuepft, die von Georg Weinberg im Jahre 1972 definiert worden ist und als Homofobie bezeichnet wird. Homofobie nimmt normalerweise verschiedene Formen an, zu denen unter anderen verbale und phisische Gewalt, die Negation im gesellschaflichen Leben und vortaeuschte, falsche Toleranz gegen LGBT Personen (Jartyś, 2016) gehoeren. Grundsaetzlich ist das besprochene Ereignis geschichtlich und stereotipisch begruendet. Es kommt auch unter der polnischen Bevoelkerung vor. Polen hat zwar im Jahre 1932 im ersten Strafgezetzbuch nach der Aneigung homosexuelle Straftat abgeschaft (Jartyś, 2016a). Der zu Koenig Polens in der Freien Elektion gewaehlte Henryk Walezy im Jahre 1573 war doch durch sein homosexuells Benehmen bekannt (Jartyś, 2016a), trotzdem gibt es in der polnischen Gesellschaft immer noch starke homofobische Erscheimngen. Auf diese Tatsache haben solche Faktoren Einfluss. Erstens ist die gesellschaftliche Erlaubnis zu keiner Toleranz derr Andersartigkeit, die ihre Herkunft in der Geschichte hat wie die dreimalige Aneigung des polnisches Staates. Auch nicht ohne Bedeutung ist der Religionskonservatismus, der als Zeichen der Nationalitaet galt, der am Ziel hatte Polen von orthodoxen Russland und und evangelischem Preussen zu unterscheiden. Drittens soll man nicht vergessen, dass das Volkspolen in den Jahren 1944-1989 paradoxal gesellschaftlich konservativ war und man keine Toleranz gegen Anderartigkeit, vor allem in der sexuellen Sphaere zeigte. Die damalige Regierung hat alles moegliche unternommen um die Nichtheterosexuelle zu deskreditieren, wie zu Beispiel unter Decknamen "Hijazinth", die von der Volkspolizei und dem Sicherheitsdienst in den Jahren 1985-1987 durchgefuerhrt wurde. Damals wurden viele Tausend homosexueller Maenner angehalten und verhoert. Man soll unterstreichen, dass diese Tatsachen die Menschenrechte und damaliges Rechtverlezt haben (Jartyś, 2016). Aber doch noch in dem Volkspolen enstand die erste illegal Organisation, die Lesben und Schwulen versamelt hat, die man als Wegbereiter in Polen fuer LGBT anerkennen kann. Sie heisst Warschauer Homosexualistenklub (WHK), der in Jahre 1987 aus der Initiative von Sławomir Starosty, Krzysztof Garwatowski und Waldemar Zboralski entstanden ist. Er wurde aber rechtlich nicht anerkannt, weil die

\footnotetext{
1 Gesellschaftliche Bewegung LGBT bezieht sich an homosexuelle Personen beider Geschlecht, bisexuelle Personen und transsexuelle. In diesen Artikel werden vom Autor Formulierungen benutzt: Nichtheterosexuelle Personen und sexuelle Minderheit beziehen sich an LGBT Personen und haben nichts gemeinsames mit dem umgangsprachlichen Verstehen der Bezeichnung sexuelle Minderheit, zu der man unter anderem Zoophihe zaehlen kann.
} 
demalige Macht seine Anmeldung abgesagt hat und begruendete auf solche Weise "Es koennten die moralische Grundlagen verletzen”. Es ist oeffentlichen dabei zu bemerken, das eben auf dieser Grundlage, der besprachenen Organisation und ihrer Mitglieder im Jahre 1989 die erste legalle Organisation fuer LGBT - erwaehnte schon "Lambda" entstanden ist (Kochanowski, 2007).

In diesem Artikel wurde Taetigkeitanalyse der Kampagne Gegen Homofobie anhand der ergriffenen Texte Internetguellen durchgefuehrt. Es werden auch Elemente der historischen Methode angewandt um das ganze Bild der Kampagne Gegen Homofobie von 2001 zu zeigen und politische Bedingungen hervorzuheben in denen fuenfzehn Jahre die Organisation taetig war. Einer von den Aspekten der Aktivitaet der gesellschaftlischen Bewegung ist sicher Kontex von Ort und Zeit, in dem sie entstehen und taetigen soll. Sie muss einen guten gesellschatlichen Hintergrund haben, von den die Ziele, der gesellschatlichen Bewegung, Ideologie an der basiert wird, und auch Formen der Mobilitaet und Handeln determiniert werden. Nicht ohne Bedeutung bleibt der politische Kontext, der in dem Moment die Entwicklung der Bewegung staerkt und unterstuetzt. Organisatorische und institutionale Formen sind auch nicht ohne Bedeutung (Nowosielski 2012). Alle erwaehnten Aspekte waren auch fuer die Entwiclung der polnischen Bewegung LGBT typisch und werden die Besteinteile der Kampagne Gegen Homofobie bilden. An der Wende des XX und XXI Jahrhunderts bewarb sich Polen um die Mitgliedschaft in der EU, fuer die Menschenrechte und auch Antidiskriminationspolitik gegen die Personen LGBT von grosser Bedeutung sind (Jartyś, 2007). Zweitens die postkommunistischen Linken haben damals politisch profitiert, die positive Einstellung zu LGBT gezeigt hatten. Drittens, es war neue Generation volljaehrig, die ihre berufliche und gesellschatliche Aktivitaet nach 1989 begann und war nicht so schwer mit der Politik Volkspolens belastet.Sie war offen der Welt gegenueber und auch auf die Verschiedenheit der sitlichen Seite des Lebens vorbereitet. Dazu haben auch die offenen Grenzen und visalose Touristik beigetragen, die von dem 8 April 1991 staendig wuchs. Die Polen, die den Westen Europas bereist haben, haben sich an die Anwesenheit der Personen LGBT gewoehnt.

Die Entsstehung der Kampagne Gegen Homofobie fiel mit dem groessten politischem Erfolg des Buendnisen der Sozialdemokraten zusammen. Im Jahre 2000 hat Aleksander Kwaśniewski, der aus dem Buemdnis abstammte, die Wiederwahl zum Praesidenten in dem ersten Wahlgang gewonnen (Państwowa Komisja Wyborcza, 2000) und ein Jahr spaeter hat das Buendnis der Sozialdemokraten wieder die Macht ergriffen, indem es die Parlamentwahl gewonnen hat (Państwowa Komisja Wyborcza, 2001). Waehrend der gefuehrten Parlamentkampagne 2001 hat sich das Buendnis an die Mitglieder der LGBT gerichtet, indem es versprochen hat, nach dem Sieg ihre Forderung zu realisieren, also das Gesetz ueber die Partnerschaft desselben Geschtechts zu verabschieden (Jartyś, 2015) des Wahlsieges hat es dem Buendnis der Sozialdemokraten nicht gelungen das Wahlversprechen das an die Nichtheterosexuellen gerichtet worden war zu realisieren.

Es gab ein paar verschiedener Ursachen. Eine von ihnen ist das Fehlen des politischen Willen und der Entschlossenheit um das Gesetz verabschieden zu koennen. Die Mehrkeit des damaligen Parteiaktivs der Sozialdemokraten stammte noch politisch aus der Volksrepublik Polens, wo die Rechte der LGBT Personen galten als Tabu. Die LGBT Personen wurden instrumental gesehen 
nur um die Waehlerschaft zu gewinnen und nicht in der Praxis zu realisieren. Das wird durch die Tatsache bestaetigt, dass es keine politische Entschlossenheit gab einen Koalitianten zu machen um das Gesetz in Parlament verabschieden zu koennen. Zweitens stand Polens vor dem Akzess an die EU. Die Sozialdemokaten haben als Prioritaet gehalten positives Ergebnis bei dem Beitritt in der Volksabstimmung zu erzielen, und die Frage des Partnerschaftgesetz koennte damals von der katolischen Kirche abgelehnt werden und dadurch die Unterstuetzung der Zentro-rechten verlieren. Zum Schluss der Legistaturperiode 2001-2005 hat das Buendnis zwar im Senat den Ertwurt des entsprechenden Gesetz eingereicht, aber das Handeln der Senatorin Maria Szyszkowska geschleitert worden ist. Das Wesetliche aber ist, dass dieses Problem erwaehnt worden war. Man soll auch bemerken, dass unter jungen Aktivisten der Sozialdemokraten befanden sich Verterer der LGBT Bewegung mit Robert Biedroń an der Spitze, dem zukuenftigen, jahrelangen Vorsitzender der Kampagne Gegen Homofobie und dem heutigen Praesidenten der Stadt Stolp (Słupsk) (Jartyś, 2015).

Die im Jahre 2001 entstandene Kampagne Gegen Homofobie besitzst den Status gemeinnuetziger Organisation und handelt anch dem Status, der ihre Ziele enthaelt. Man kann sie folgend charakterisieren: das Bemuehen zur Aenderung der geltenden in polnischem Rechtsystem Vorschiften, die die Diskrieminierung betreffen wegen der anderen Sexualorientierung und Geschlechtidentitaet, das Verbreiten des Wissens um der Gesellschaft die Personen mit anderen sexuellen Orientierung und ihre Familien zu akzeptieren, Instutionaliesierung im polnischen Rechsystem Partnerschaften von der Geschlecht abgesehen, Diskriminierungsverminderung im Gesundheitsystem und handeln so, um die Qualitaet der medizinischen Dienstestungen zu verbessen, Gesundheitsschutz und Gesundheitsvorberungen foerdern fuer sicheren sex werben, Gegenmassmahmen treffen, wenn es um HIVAnsteckung und AIDS Erkrankungen geht, Taetigkeitsbereich fuer Freiheit vergroessern und Diskrieminierug bekaempfen an den Schulen, Edukationsstellen und Hochschulen. Die Verbesserung der Edukaionqualitaet, die die sexuelle Verschiedenheit betrifft, Geschlechsidemtitaet und sexuallen Expression in allen Stufen der Edukation, unterstuetzung der Resozialisationprozesse und Einfluss auf die Erziehung der jungen Generation, Einfuehrung zu den polnischen Rechtsystem solcher Vorschriften, die die Verbrechen, die Hass und Intoleranz poenalisiert werden, groessere Untersstuelzung fuer die Opfer der Gewalt, Arbeiten zahlloser Berantungen - psychologischen, juristischen und sexuologischen Hilfe fuer die Familien der LGBT ihre Naechsten, die Informationen verbreiten uber Kinderrechte, Gesundheitsrechte und Reproduktionrechte, Unterstuezung der europaechen Integration, Demokratie, Buergerfreiheiten und und Staerkung der patrotischen buergelischen Haltung (Kampania Przeciw Homofobii, 2016).

Die Realisierung ober erwaehter Ziele Kampagne Gegen Homofobie nimmt verschiedene Formen an und hat intensiven Character. Bisherige massnahmen wurden von der Organisation auf hundertdreiundsechzig Internetseiten angehaengt. Nach dem Feststellung der Kampagne Gegen Homofobie, ihrer Taetigkeitbereich in den leztten 15 Jahren, und Aktiwietaet0organisierung haben dazu gefuert, dass die Zustimmung fuer die LGBT Personen um 15 Prozent gestiegen ist (Kampania Przeciw Homofobii 2016). 
Es lohnt sich zu bemerken, das die Taetigkeit der Kampange Gegen Homofobie viele Aspekten hat. Sie ist sowohl an die LGBT Personen als auch an die ganze Gesellschaft gerichtet. Man kann sie also einzelne Elemente teilen. Einer von ihnen ist Informationwissenschaftlicher Taetigkeitbereich, der zyklische Raporte ueber die Situation LGBT Personen in Polen herausbibt, und zeigt seit 2002 Gefahrlage und homofobisches Verhalten in der Politik und im Gesellschaftsleben. Man kann soll dabei zeigen, dass mit der Entwiclung Kampagne Gegen Homofobie die Raporte immer groesseren Volumen haben und schildern Gefahraspekte im geselschaftlichem Leben stellen die Beziehung zu KGBT Personen in der Administration, bei der Polizei, im dem Schulwesen im Gesundheitsdienst dar. Im gesellschaftlichen Raum wird auch die Groesse der physsichen und psychischen Gewalt gegen LGBT Personen gezeigt. Die in den Dokumenten erhaltene Informationen stellen homofobische Aussagen der Vertreter des politischen Lebens in Polen dar, obwohl sie fuer Freude der LGBT Personen annerkannt werden moechten. In der Rapporten aus den letzten fuenfzehn Jahren kann man die Angabe der damaligen Abgeordneten Anita Błochowiak aus dem Buendnis der Sozialdemokraten finden, in der sie sich ueber Schwuler, die rote Socken an hatten geaessert hat. Und dann hat Ewa Kopacz, Robert Biedroń wegen seiner sexuellen Orientierung zur Zeit der Kommunalwahler 2002. Die darggestellten Verhalten bestaetigen definitiv die Homofobie, von der ich in dem ersten Teil meines Artikels geschriebien habe. Sie bestaetigen so genannte falsche Toleranz gegen die Nichtheterosexuellen (Jartyś, 2016).

Manche von den Beispielen des unkorekten Bemehmens gegen LGBT Personen in Polen von denen immer wieder laut war, erhielten positive Resultate. Beispielweise, KGH fuerte Kampagne fuer Blut, deren Aufgabe war das Abschafen von Aufzeichnnung, die mit Blutspender und Blutsprenderstellen der Homoseksuallen vor allen Maenner verbunden war. Das Endergebnis der Gespraeche mit Minister Bartosz Arłukowicz vom 31 Dezember 2009 (Rozporządzenie, 2009) war neue Verordnung, die die Blutabmahme voellig veraendert hat. Die Formulierung, dass die homosexuellen Maenner disqualifiziert sind, wurde durch neue ersetzt, dass die wegen ihrer homosexuelln Verhalten zur Gruppe der Blutsprender mit hoeherem Ansteckerisiko gehoeren, wenn es um schwere Krankenheiten beim den Bluttransfusion geht. Die neue Ministerverordnung betrifft alle Personen unabhoenging von der sexuellen Orientierung. Sie ist also mit der Rechlinie der Europaeischen Kommision vom 27 Januar 2003 uebereinstimmend, die mit der Signature 2002/98EC (Directive, 2003) gekennzeichnet wird. Das bedeutet, dass die Prinzipien der gefahrlosen Blutabgabe mit der sexuellen Orientierung nichts zu tun haben (Jartyś, Bulsa, 2015).

Ausser der zyklisch herausgegeben Rapporten soll man auch anderen Publikationen der KGH Aufmeksamkeit schenken. Es geht hier um juristische, psychologische, gesundheitsfoermdernde und bildende Veroeffentlichungen. Man kann ueber ihrer Rechtsprechung des polnischen Gerichts finden das die Rechte der LGBT Personen betreffen Publikationen der gesundheitsfoernderungen Politik und HIV - Gegenmassnahmen, AIDS -Erkrankungen, aber auch Geschichte der Verfolgung der LGBT Personen im Hitler-Deutschland in den Jahren 1933-1945. Die Publikationen, die von der Kampagne Gegen Homofobie signatiert werden, bilden interessanten Untersuchungsmaterial fuer die Leute, die sich breit und weit mit Sozialwissenschaften befassen und untersuchen die gesellschaftliche Bewegung LGBT in Polen. Gleichzeitung bilden die 
erwaehnten Publikationen eine Wissensquelle fuer die Nichthomosexuellen, ueber ihre Rechte und gesundheitsfoerdernde Lebendweise. Die Organisation steht von Anfang an fuer die Hilfe und Verfuegung in allen wissenschaftlichen Untersuchungen, die die gesellschaftliche LGBT Bewegung betreffen. Deshalb fuert sie eigene Bibliothek, deren Sammlungen allen, die interessiert sind zur Verfuegung sind, auch als Postsendungen. Die Buechersammlungen werden sukcesiv vergroessert und enthalteng ausserdem Dokumente, Monograhien und Zeitschriften, die die Nichheterosexuelle betreffen (Kampania Przeciw Homofobii, 2016).

Die Bibliothekarische Taetigkeit KGH wird von ihr selbst als eine Art auf dem Forum, die Hilfe leistet, die sie unternimmt. Ausser ihr von grossen Bedeutung ist die psychologische und juristische Hilfe, die sie leistet. Wenn es um juristische Beratung geht unterstuetzt die KGH die Personen, die diskriminiert werden und deren persoenlische gueter wegen ihrer Orientierung verletzt werden. Jeder Geschaedigete kann per mail, oder bei frueher gemeldeten Treffen von den $\mathrm{KGH}$ Juristen beraten und gefuert werden. Es ist bemerkenswert, dass auf diesem Gebiet KGH grosse Erfolge erzielt hat. Sie war sehr stark angagiert in die Angelegenheit von Ryszard Giersz hat seine Nachbarin Dorota Sz. von Gericht gestellt und hat in beiden Instanzen zufriedende Entscheidung bekommen. Dorota Sz. bekam eine Geldstrafe indem sie geaussert hatte, da $\beta$ sie ihn mit schlimmen Worten beschimpft hatte, die allgemein fuer jeden beleidigend sind. Sie hat damit seine Wuerde und Lebenschutz verletzt. KGH hat ihre Zufrienerheit geaessert, und in ihrem Namen hat sich Robert Biedroń geaeussert: "Fuer uns ist es ein wichtiger Urteil. Es war kein Kampf um Geld, aber um Wuerde. Es geht nicht um einzelnen Menschen, sondern um alle Homosexuellen. Wir sind ihm dankbar fuer seinen Mut, dafuer, das ser sein Gesicht gezeigt hat und ganze Erniedrigung ueberwindet hat. Das Erniedrigen der Lesben und Schwulen ist in Polen üblich, aber ich glaube, dass wir jetzt von keinem nicht mehr, als Leute der zweiten Kategorie behandelt werden. Die Gesellschaft auch" (Jartyś, 2015a, s. 118).

$\mathrm{Zu}$ wesentlichen und wichtigen Faetigkeitsaspekten der KGH ist die psychologische Hilfe, die den Personen und ihren Familien erteilt wird. Sie ist unentbehrlich in Falle der muendlicher Gewalt und psychischer wegen der sexuellen Orientierung, das Quaelen in der Schul - Hochschulumgebung, am Arbeitsplatz und im Falle fehlender Selbstakzeptierung und Schwierigkeiten bei Verstehen eigener Sexualorientierung. Die erwaehnte Unterstuetzungsform ist fuer die Nichtheterosexuellen besonders wichtig aus vielen Ursachen. Erstens kann sich nicht jeder selbst helfen, wenn er die Gewalt erlebte auf dem Grund seiner sexuellen Orientierung. Die Raportten der KGH erscheinen seit 2002 und zeigen das Spektrum der muendlichen und physischen Gewalt, die die LGBT Personen in unserem Land erlitten haben. Sehr oft kommt es vor, dass die Opfer vor Angst melden das Ereignis nicht bei der Polizei. Sie rechnen dabei mit Verpoennung und Verstaendnislasen Polizeibeanten. Aehnliches Problem kommt bei dem Besuch des Gesundheitsdienstes, wo die Nichtheterosexuelle Person fuehlt Furcht, wenn sie ueber intimme Angelegenheiten bei Frauenarzt oder Venerologenbesuch sprechen soll und muss bestehen, dass sie Homosexuell ist (Jartyś 2015a). Man soll bemerken, dass unter veilen Personen LGBT psychologische Staerkung unentbehrlich ist um so genannte kleinere Furcht zu minimalisieren, sie jede Minderheit begleitet und von der gesellschaftlichen Natur aus, tritt bei LGBT Personen 
stark auf. Die naechste von den Medien nicht beruehrte Angelegenheit, die KGH Hilfe leistet ist das Akzentieren der Nichtheterosexuellen Person im Familienhaus. Es kommt oft vor, dass die Eltern finden keine Nachsicht fuer sexuelle Orientierung ihres Kindes, abgesehen davon, ob es Jugendliche, oder schon Erwachsene ist. Zum Schluss werden die Beziehungen abgebrochen, zu Hause gibt es Schikane und dann Ergebnis - das Familienhaus muss verlassen werden. Der Autor hatte auch mit solchen Faellen zu tun, als er Untersuchungen ueber Aktivitaet und Sicherheit der LGBT Personen im Internet analysierte. Er fuehrte auch Konversationen auf dem Portal - gesellschaftlichen Facebook. Im Rahmen der psychologischen Hilfe, die dank der Vermittlung KGH stattfindet, wurde Unterstuetzungnetz geschaffen, der Kontakte mit den Psychologen in solchen Staedten wie Warszawa, Łódź, Pruszków, Katowice, Gdańsk, Sopot, Szczecin, Chrzanów, Legnica, Wrocław, Jelenia Góra, Tarnowskie Góry, Bytom, Poznań, Kraków i Bydgoszcz (Kampania Przeciw Homofobii, 2016).

In ihrer bisherigen Faetigkeit hat KGH initiert und mitgeschafft viele Vorstellungen, bei denen die KGH Personen im gesellschaftlichen Raum anwesend waren. Seit Anfang an haben ihre Vertreter jedes Jahr an den Paraden und Marschen, die in den Strassen Warszau und anderen grossen Staedte stattfanden teilgenommen. KGH hatte ihre Unzufriedenheit gegen Verbot, den genannten schon Manifestationen, die von den Machtorganen der Gemeinde - und Bezirks selbstverwaltung in den Jahren 2005-2007 stattgefunden haben. Im Endergebnis der damals gemachter Muehe, dank Medien und internationalisiertem Problem tauchte die Frage auf, wo liegen die Freiheitsgrenze des Manifestierens in Polen. Auf den eingebrachten Vorschlag von dem damaligen Buergerrechtsbeauftragten Prof.Andrzej Zoll hat der Verassunggerichtshof unter der Leitung Prof. Eva Łętowska auf der Sitzung am 18. Januar 2006 die Manifestierenfreiheit auf dem Gebiet Polens anerkannt. Er hat noch darueberhinaus hingewiesen, dass die Gemeindeselbstverwaltungen sie nicht begrenzen koennen, indem sie sich auf die ideologische Voraussetzungen oder das Sicherheitsfehlen fuer die Teilnehmer des Zusammenkommens berufen, weil Sicherheitsgarantie ihre Aufgabe ist. Man kann vermuten, dass dank der LGBT Bewegung wurden in Polen die Prinzipien der Zusammenkommenfreiheit bestimmt, die allen Polenbuergern zu nutzen stehen, hier auch den Gegenern der gesellschaftlichen Bewegung (Jartyś, 2015).

Ueber bisherige fuenfzehnjaehrige Taetigkeitszeit hat die KGH ein paar Aktionen und Medienkampagne durchgefuehrt, die am Ziel hatten, der Bevoelkerung bewusst machen, dass hier auch die Nichtheterosexuelle Personen leben, die oft Kolleginnen und Kollegen am Arbeitsplatz sind, oder Nachbarn, oder Bekannte. Weil der Antor vorliegenden Artikel umfangsmaessig begrenzt ist, moechte er nur ein paar Aktionen, die von KGH an die LGBT Personen und an die Gesellschaft gerichtet sind. Es ist bemerkenswert, dass die Menge der durchgefuehrten Aktionen und ergriffen Taetigkeitsformen erlauben eine getrennte Publikation zu bearbeiten. Als erste war die Aktion unter Motto "Sie sollen uns sehen", die im Jahre 2003 durchgefuehrt wurde. In den Kunstgalerien und auf Billboarden, Werbeplakaten in groesseren Staedten Polens konnte man Gesichte von 30 homosexuellen sehen, die Hand in Hand gehalten haben. Auch um Jahreswende 2003/2004 hat KGH eine Aktion durchgefuehrt unter dem Motto: "Ich bin Schwule, ich bin Lesbe. Lerne uns kennen!” Damit wollten sie eben Homosexualismus zeigen. Die Schoepfer 
dieser Kampagne waren dessen bewusst, dass das Wissen darueber in unserer Gesellschaft miseraber ist und dass man daran stereatypisch denkt. Darueberhinaus wird nicht selten der Homosexualismus als eine Krankheit oder psychische Verwirrung begriffen, was im Gegensatz zu medizinischen Standpunkt steht. Also vom medizinischem Standpunkt aus, ist Homosexualismus keine Krankheit seit 1991. So hat Gesundheitsweltorganisation entschieden. Die von KGH Aktion wurde an die Akademiker und Lehrer und Eltern der Nichtheterosexuellen gerichtet. In ihrer Dauerzeit hat man an den Hochschulen darueber debatiert unter anderem in Warszawa, Lublin, Białystok, Gdańsk, Kraków, Poznań, Wrocław, Łódź und Katowice. Einer von den Aspekten des durchgefuehren Unternehmens wurde die Moeglichkeit sich mit den Psychologen und Sexuologen zu treffen und sich beraten zu lassen, weil andere sexuelle Orientierung oft Generationskonflikte vernsacht. Die von der KGH Aktion war mit dem Ratgeber, der an die Eltern der LGBT Personen gerichtet war, der im Jahre 2005 veroeffentlich wurde. Zewi Jahre spaeter wurde naechste Aktion durchgefuehrt "Du bist nicht allein", die am Ziel hatte LGBT - Milien $\mathrm{zu}$ integrieren und die Personen, die commingot befuerchtet haben zu unterstuetzen, also in der Oeffentlichkeit ihre sexuelle Orientierung zu zeigen. In dem Drei - staedtenverbund (Gdańsk, Gdynia, Sopot) und in Toruń wurde Reklameblaetteraktion durgefuehrt - auf 93 Saeulen wurden 500 Plakate befestigt. Nicht alle Vorhaben der KGH wurden mit Entusiasmus durch die ganze Gesellschaft willkommen z.B. eine von den Reklamefirmen aus Bydgoszcz ist zurueckgetreten, den wahrscheinlich wollte nicht mit der Bestellung fuer die LGBT assoziert werden (Leszczuk-Fiedziułkiewicz, 2014). Auch die unternommen Aktion "ueber - reichn wir uns das Friedenzeichen" im Jahre 2016, die am Ziel hatte zu zeigen, dass von den christlichen Werten die Notwendigkeit der Hochachtung, ofenheit und des freundlichen Gespraechs kommt, wird in manchen Katholischen Kreisen kritisch angenommen (Episkopat: katolik nie powinien..., 2016).

KGH und ihr Handeln wecken im der polnischen Gesellschaft aeusserste, extreme Emotionen. Die Anhaenger finden ihre Taetigkeit gut und betonen die Offenheit, die Arbeit fuer Buergergesellschaft, des Aufrufen zur Toleranz gegen LGBT Personen in Polen. Die Gegner sehen in der KGH eine Organisation, die fuer Homosexualismus wirbt und steht im Konflikt zu dem traditionellen Modell der Familie. Die verschiedene Beuerteilung kommt aber aus der Tatsache, dass sie KGH nimmt unbekannte Probleme auf, die von der polnischen Gesellsacht noch immer fern sind und grundsaetzlich sehr kleine Interesse an den Nichtheterosexuellenrechten zeigt. Wenn man aber im Grossen und Ganzen alle Tatsachen, die gelungen sind analysiert, muss man feststellen, dass ihre Taetigkeit positiv in grossen Teil der Bevoelkerung angenommen wird. Es ist auch schwer festzustellen, ob der jahrelang an der spitze der KGH stehende Robert Biedroń den Wahlsieg erzielt haette bei den Parlamentarwahlen 2011 und dann im Jahre 2014 zum Praesidenten der Stadt Słupsk wurde, ohne frueher bei der LGBT Bewegung gearbeitet zu haben. Diese Arbeit hat ihn bekannt gemacht. Er ist bei der Bevoelkerung als informeller, unbestrittener Anfuehrer der polnischen LGBT Bewegung geworden, dazu jahrelange Arbeit der KGH beigetragen hat. 


\section{Bibliografie}

Arzneimittel, die für Prüfungen in Forschung und Entwicklung bestimmt sind, werden von der Richtlinie 2001/83/EG des Europäischen Parlaments und des Rates [5] nicht erfasst.

Directive 2002/98/EC of the European Parliament and of the Council of 27 January 2003 setting standards of quality of safety for the collection resting, processing storage and distribution of human blood and blood components and amending Directive 2001/83/EC. Official Journalof the European Union, 08.02.2003: 33-38.

Jartyś, J. (2015a). Bezpieczeństwo społeczne osób Lesbian, Gay, Bisexual, Transgender (LGBT) w Polsce. In: M. Cupryjak, P. Nowak (Hrsg.), Wybrane zagadnienia współczesnego bezpieczeństwa społecznego. Szczecin: Wydawnictwo Volumina.

Jartyś, J. (2016). Homofobia w polskiej przestrzeni społecznej i politycznej. In: F. Pierzchalski, J. Golinowski (Hrsg.), Socjotechnika lęku w polityce. Bydgoszcz: Wydawnictwo Uniwersytetu Kazimierza Wielkiego.

Jartyś, J. (2016a). Przestrzeganie europejskich standardów praw człowieka wobec osób Lesbian, Gay, Bisexual, Transgender (LGBT) w Polsce i na Ukrainie. Analiza porównawcza. In: K. Sygidus, O. Gorbach u.a. (Hrsg.), Ukraina. Konflikt, transformacja, integracja. Olsztyn-Lwów: Wydawnictwo Sroka.

Jartyś, J. (2015). Wkład Polski do procesu umiędzynarodowienia i rozwoju praw osób Gay, Lesbian, Bisekxual, Transgender (LGBT) po akcesji Polski do Unii Europejskiej. In: L. Wojnicz, D. Rdzanek, M. Potkańska (Hrsg.), W poszukiwaniu finalite politique Unii Europejskiej. Aspekty teoretyczne i praktyczne. Szczecin-Warszawa: Instytut Politologii i Europeistyki Uniwersytetu Szczecińskiego, Instytut Europeistyki Uniwersytetu Warszawskiego.

Jartyś, J. (2016). Udział kandydatów Lesbian, Gay, Bisexual, Transgender (LGBT) w wyborach samorządowych 2014 roku w Polsce. In: K. Kamińska-Korolczuk, M. Mielewczyk, R. Ożarowski (Hrsg.), Między teoria a praktyka funkcjonowania systemów politycznych i partyjnych. Systemy polityczne i partyjne w ujęciu teoretycznym i praktycznym. Gdańsk: Uniwersytet Gdański, Wydział Nauk Społecznych, Instytut Politologii.

Jartyś, J. (2007). Wybrane aspekty przestrzegania europejskich standardów praw człowieka wobec gejów i lesbijek w Polsce. In: J. Jartyś, A. Staszczyk (Hrsg.), Demokratyzacja i prawa człowieka w Europie Środkowo-Wschodniej.Szczecin: Wydawnictwo Zapol.

Jartyś, J., Bulsa, M. (2015). Problem krwiodawstwa i krwiolecznictwa w raportach polskiego ruchu Lesbian, Gay, Bisexual, Transgerder (LGBT) w Polsce. Hygeia Public Health, 50 (1), 80-83.

Kampania Przeciw Homofobii (2016). Herunterladen: www.kph.org (28.10.2016).

Kochanowski, J. (2007). Poza horyzont hetero normatywności. Ruchy społeczne gejów i lesbijek. Herunterladen: www. ekologiaisztuka.plthink.tankfeministyczny/kurs/kochanowski2007 (28.10.2016).

Leszczuk-Fiedziułkiewicz, A. (2014). Medialny obraz związków partnerskich w polskich kampaniach społecznych w latach 2004-2013. Niscellanea Historico-Juridica, XIII (2), 353-387.

Nowosielski, M. (2012). Ruchy społeczne jako czynnik i wynik zmian społecznych. Kultura i Edukacja, 4 (90), 7-33.

Państwowa Komisja Wyborcza (2000). Wybory Prezydenta Rzeczypospolitej Polskiej 200. Wyniki oficjalne. Herunterladen: http://prezydent2000.pkw.gov.pl/wb/wb.html (28.10.2016).

Państwowa Komisja Wyborcza (2001). Wybory do Sejmu: wyniki głosowania na listy komitetów wyborczych w skali kraju. Herunterladen: http://wybory2001.pkw.gov.pl/sjg2_k.html (28.10.2016).

Rozporządzenie Ministra Zdrowia z dnia 31 grudnia 2009 r. zmieniające rozporządzenie w sprawie pobierania krwi od kandydatów na dawców krwi i dawców krwi. Dz.U. RP, nr 7, poz. 50.

Episkopat: katolik nie powinien brać udziału w kampanii „Przekażmy sobie znak pokoju” (2016). Herunterladen: http://www.tvn24.pl/wiadomosci-z-kraju,3/prezydium-kep-akcja-przekazmy-sobie-znak-pokoju-nie-dlakatolikow,676228.html (28.10.2016). 
